\title{
COMMENTARY
}

\section{Care bundles: implementing evidence or common sense?}

\author{
Luigi Camporota and Stephen Brett* \\ See related research by Niël-Weise et al., http://ccforum.com/content/15/2/R111
}

\begin{abstract}
Care bundles aim to improve standard of care and patient outcome by promoting the consistent implementation of a group of effective interventions. However, a variety of barriers prevent their full application in clinical practice. Here, we discuss some of the benefits and limitations of care bundles in the delivery of safer and more effective and consistent health care.
\end{abstract}

In things necessary, unity; in things doubtful, liberty.

St. Augustine

In the previous issue of Critical Care, Niël-Weise and colleagues [1] investigate the effectiveness of the semiupright position (and elements of the 'ventilator bundle') in preventing ventilator-associated pneumonia (VAP).

The effective and consistent implementation of beneficial treatments can improve patient outcome. However, while physicians try to reconcile the art with the science of medicine by attempting to apply at the bedside the results of clinical trials, intensive care patients receive, on average, only about one half of the recommended core clinical interventions.

This inconsistency in clinical decision-making and care provision is not due exclusively to variation in case mix and facilities but is the result of the intrinsic complexity of critical care, on one hand, and the physician's heuristics (that is, intuitive judgments) on the other [2]. Errors of omission, such as failure to perform routine tasks or an intended plan, or the loss of key information

*Correspondence: stephen.brett@imperial.ac.uk

Centre for Perioperative Medicine and Critical Care Research, Department of Anaesthesia and Intensive Care, Hammersmith Hospital, Imperial College Healthcare NHS Trust, Du Cane Road, London, W12 OHS, UK at handover [3] are particularly relevant today, when the management of critically ill patients is more complex, the working shift pattern of health-care professionals requires multiple daily handovers, and patients and physicians experience discontinuity in care. These considerations highlight the fact that idiosyncratic practices are unsustainable and dangerous. The standardization of the process of care, therefore, requires structuring the task environment and compensating for heuristics to facilitate consistent and reliable delivery of best evidencebased practice. This is the aim of care bundles [4], defined by the Institute of Healthcare Improvement as a 'a group of interventions related to a disease process that, when executed together, result in better outcomes than when implemented individually' [5]. The definition implies that its elements should function as a package and that its effectiveness comes from the excellence of the supporting evidence and its consistent comprehensive execution.

Although proponents of standardized, protocol-driven care see the conceptual advantages of bundles (that is, that they simplify decisions, reduce omissions and errors in medical reasoning, promote goal-orientated care, and deal with areas of uncertainty by giving a pragmatic but consistent solution) [4], adherence to bundles depends on the interplay between factors that act as barriers or enablers. Commonly, lack of knowledge, unavailability of resources, high costs, nursing convenience, fear of adverse events or patient discomfort, and (most importantly) disagreement on the strength of the supporting evidence [6] in conjunction with external barriers can affect a physician's ability to execute recommendations [7]. In this context, Niël-Weise and colleagues [1] report a systematic review and the recommendations of a European expert panel on the benefits and disadvantages of the semi-upright position in the prevention of VAP.

The authors found nonsignificant reductions in the incidence of VAP (clinically suspected and microbiologically confirmed) and in mortality with semi-upright position with no sufficient data to quantify harm (for example, venous thromboembolism, hemodynamic instability, or patients' discomfort) [1]. In this context, the 
expert panel suggested that the semi-upright position be used as the preferred option but only in the absence of clear contraindications (for example, spinal injury) and with necessary restrictions (for example, nursing tasks, medical interventions, and patients' wishes) [1].

Expert opinions and adherence to recommendations often seem dissociated from the strength of clinical evidence. For instance, some strategies considered to be ineffective by trials have high rates of adherence, whereas others found to be effective (for example, continuous subglottic aspiration and digestive decontamination) have a high rate of nonadherence [6].

In addition, regardless of (or, occasionally, despite) the strength of evidence, some clinicians oppose the concept of care bundles in general, arguing that bundles (a) are used by industries as a marketing tool, (b) deprive clinicians of clinical autonomy, (c) are inefficient (similar benefit could be achieved with fewer elements) or ineffective as they may divert from the implementation of a more effective set of interventions not included in the bundle, (d) increase the risk of over- or under-treatment (that is, not all patients need all elements of the bundle all of the time), (e) may be inappropriately adopted as a measure of organizational performance [8], or (f) suffer from positive publication bias and lack of external validity in 'the real world' or contain elements that are not plausibly related to the bundle's objectives (for example, thromboprophylaxis and VAP prevention).

In the paper by Niël-Weise and colleagues, the expert panel recommended upright head elevation, despite a lack of strong supporting evidence [1], as did the UK National Institute for Health and Clinical Excellence (NICE guidance PSG002) [9]. This apparent discrepancy between 'evidence-based' and 'common-sense' recommendations may reinforce the perception that committees' advice is 'unscientific' or attempts to drive consensus rather than reflect it $[10,11]$.

Given these uncertainties, the question for the practicing clinician is, should we use bundles and protocols? In the presence of clear evidence, the answer seems simple: if the components are scientifically sound, yes; but when the evidence is conflicting, a common-sense approach is necessary. The best guess involves the consistent use of protocols for routine and common practice, as overall they are unlikely to cause significant harm and are more likely to be beneficial. However, the optimal balance between protocolized versus individualized care will change among institutions, depending on staffing and case mix [3], and with the availability of new research. In this context, using compliance to each element of a bundle as an indicator of performance may not reflect quality of care unless other considerations of risks and benefit of the proposed interventions are included and the reasons for deviation are reported. In the meantime, we should strive to implement current performance systems to deliver the consistent engineered care that patients and their families expect and deserve.

\section{Abbreviations}

VAP, ventilator-associated pneumonia.

\section{Competing interests}

The authors declare that they have no competing interests.

Published: 24 May 2011

References

1. Niël-Weise BS, Gastmeier P, Kola A, Vonberg RP, Wille JC, van den Broek PJ, Bed Head Elevation Study Group T: An evidence-based recommendation on bed head elevation for mechanically ventilated patients. Crit Care 2011, 15:R111.

2. Mohan D, Angus DC: Thought outside the box: intensive care unit freakonomics and decision making in the intensive care unit. Crit Care Med 2010, 38 (10 Suppl):S637-641

3. Walker S, Brett S: Oiling the wheels of intensive care to reduce'machine friction': the best way to improve outcomes? Crit Care Med 2010, 38 (10 Suppl):S642-648.

4. Rello J, Chastre J, Cornaglia G, Masterton R: A European care bundle for management of ventilator-associated pneumonia. J Crit Care 2011, 26:3-10.

5. Institute for Healthcare Improvement: Sepsis [http://www.ihi.org//HI/ Topics/CriticalCare/Sepsis/].

6. Rello J, Lorente C, Bodi M, Diaz E, Ricart M, Kollef MH: Why do physicians not follow evidence-based guidelines for preventing ventilator-associated pneumonia?: a survey based on the opinions of an international panel of intensivists. Chest 2002, 122:656-661.

7. Cabana MD, Rand CS, Powe NR, Wu AW, Wilson MH, Abboud PA, Rubin HR: Why don't physicians follow clinical practice guidelines? A framework for improvement. JAMA 1999, 282:1458-1465.

8. Amerling R, Winchester JF, Ronco C: Guidelines have done more harm than good. Blood Purif 2008, 26:73-76.

9. Technical patient safety solutions for prevention of ventilator-associated pneumonia in adults: understanding NICE guidance [http://guidance.nice. org.uk/PSG002/Publiclnfo/doc/English].

10. Antonelli M, Mercurio G: The 2008 international guidelines for management of severe sepsis and septic shock: merits and weaknesses. Minerva Anestesiol 2009, 75:27-29.

11. Reade MC, Warrillow SJ, Myburgh JA, Bellomo R: Guidance in sepsis management: navigating uncharted waters? Crit Care 2008, 12:428; author reply 428 .

\section{doi:10.1186/cc10232}

Cite this article as: Camporota L, Brett S: Care bundles: implementing

evidence or common sense? Critical Care 2011, 15:159. 